\title{
Comparación de desempeño de observadores de estado en sistemas lineales con aplicación a un motor de corriente continua
}

\section{Performance comparison of state observers in linear systems through the application to a continuous current engine}

\author{
Alexander Eduardo Florián Villa \\ Gestor de Proyectos, Tecnoparque SENA Nodo Medellín -Grupo GIGAT, Medellín, Colombia. aflorian@sena.edu.co
}

Recibido: mayo 22, 2018. Aceptado: junio 30, 2018.

\begin{abstract}
Resumen
En muchos procesos industriales existen variables importantes desde el punto de vista de control de procesos que requieren de vigilancia y monitoreo constante; sin embargo, muchas veces es una tarea de gran dificultad o simplemente no existe un sensor adecuado para realizar esta medición. Esto genera un gran reto para los ingenieros quienes deben, de alguna manera, tener medición sobre las variables del sistema. Una alternativa cuando no se tiene acceso completo al vector de estados del sistema, es obtener una estimación de los estados no medibles a través de un observador de estados, sistema dinámico cuyos estados convergen a los del sistema observado, basados en el modelo del sistema y las medidas disponibles de la entrada y la salida de este. Estos observadores sirven para estimar variables que sean difíciles de medir, de tal manera que se pueda describir el comportamiento del sistema dinámico. En este trabajo se prueban una serie de observadores de estado para estimar la velocidad de un motor de corriente continua (DC). Utilizando diferentes estimadores de estados tales como el Estimador Luenberger, el Filtro de Kalman para sistemas lineales y el Observador por Modos Deslizantes se pretende estimar correctamente la velocidad del motor DC partiendo de su modelo matemático y la medición de corriente del estator. Tanto en la implementación como en la simulación de los observadores, se observa que las perturbaciones inesperadas podrían alterar el comportamiento del sistema. Los resultados apuntan a que cada observador tiene unas condiciones específicas en las que su desempeño es más efectivo
\end{abstract}

Palabras Clave: Sistema, modelo, estado, vector de estado, observabilidad, dinámica, estimador, medición.

\begin{abstract}
In many industrial processes, there are essential variables which need constant monitoring for process control tasks. However, often such measurement is complicated, or there is just no adequate sensor to perform this goal; this creates a significant challenge for engineers, who must somehow have measurement and control over system variables. An alternative for cases in which the access to the state vector is unfeasible is to obtain an estimate of the non-measurable states of the system through a state observer. The observer is a dynamic system whose states converge to those of the observed system, based on the system model and the observation of the input and output of the system. This information is used to estimate variables that are difficult to measure to make possible the full description of the behavior of the dynamic system. In this work, a set of state observers is designed and implemented to estimate the speed of a DC motor by measuring its stator current. Using different types of state estimators such as Luenberger Estimator, Kalman Filter for Linear Systems and the Sliding Mode Observer, we intend to estimate the DC motor speed correctly based on its mathematical model and stator current measurement. In both the simulations and the implementation of the observers, the response of these to unexpected perturbations that could alter the standard conditions of the system is analyzed and compared. The results suggest that each observer has specific conditions where their performance is enhanced.
\end{abstract}

Keywords: System, model, state, state vector, observability, dynamics, estimator, measurement

\section{INTRODUCCIÓN}

Un observador es un algoritmo utilizado para estimar las variables de estado de un sistema dinámico [1]. Además, los observadores de estado poseen importantes propiedades adicionales tales como la posibilidad de contar con una secuencia estructurada de diseño y la capacidad de utilizar dinámicas reducidas para la observación del proceso en cuestión [2]. En general, varios investigadores se han interesado por el diseño de observadores de estado y se encuentran varias soluciones reportadas en la literatura aplicadas a problemas de estimación, identificación de modelos y diseño de controladores, entre otros [3]-[11].

Citar como:

A.E. Florián-Villa. “Comparación de desempeño de observadores de estado en sistemas lineales con aplicación a un motor de corriente continua" Revista CINTEX, Vol. 23(1), pp. 5159. 2018. 
Comparación de desempeño de observadores de estado en sistemas lineales con aplicación a un motor de corriente continua Performance comparison of state observers in linear systems through the application to a continuous current engine

En este documento se muestra que con el modelo del sistema y la observación de las variables de entrada y salida se puede reconstruir el vector de estado que describe completamente la dinámica del sistema y su comportamiento. En la figura (1) se presenta un diagrama general del observador de estado en un sistema dinámico, en el que se aprecia la relación existente entre las variables de entrada, salida y el modelo del sistema, elementos necesarios para la implementación de un observador de estados [12].

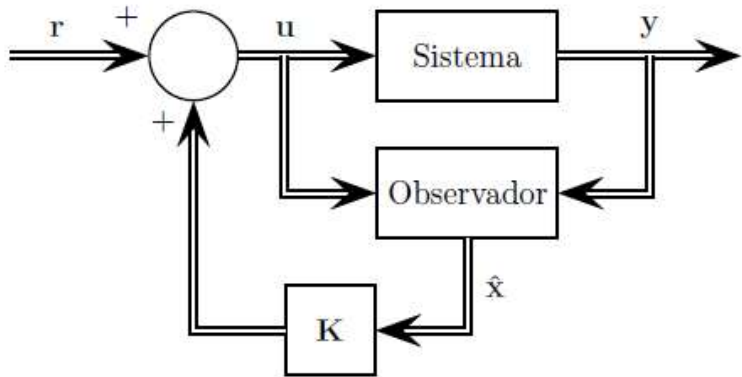

Figura 1. Diagrama de realimentación por variables de estado con observador. Elaboración propia con base en [13].

El concepto de observabilidad de un sistema está asociado a la posibilidad de determinar el estado del sistema a partir de las mediciones de sus entradas y salidas durante un tiempo finito, es decir, que si este criterio se cumple existe la posibilidad de reconstruir el estado del sistema a partir de un observador de estado. Lo anterior indica que la inspección y el cumplimiento del criterio de observabilidad del sistema es fundamental para que el observador de estados converja correctamente a los estados del sistema observado; el problema radica en encontrar condiciones en las que un conocimiento de los datos de entrada-salida reconstruya la forma única del estado del sistema. Consideramos el sistema lineal mostrado a continuación [14], donde $x \in R^{n}, u \in R^{m}$ y la variable $y \in R^{p}$ :

$$
\begin{gathered}
\dot{x}=A x+B u \\
y=C x
\end{gathered}
$$

En la ecuación anterior, la matriz $A$ se define como matriz de estados, la matriz $B$ se define como matriz de transición y la matriz $C$ como matriz de salida, estas matrices se asumen conocidas. La ecuación de estado (1) se denomina como observable si para cualquier estado inicial $x(0)$ (desconocido), existe un tiempo finito $t_{1}$ tal que el conocimiento de la entrada $u$ y la salida $y$ sobre el intervalo $[0 ; t 1]$ es suficiente para determinar en forma única el estado inicial $x(0)$, en caso contrario el sistema es no observable. Debe ser posible determinar el estado inicial a partir de cualquier tiempo final [15].

Kalman demostró que la observabilidad podía analizarse fácilmente empleando un test que actualmente se denomina test de observabilidad de Kalman, el cual establece que si la matriz $M_{0}$ (ver ecuación 2) es de rango $n$ igual a la dimensión del sistema, el sistema es observable [16]:

$$
M_{0}=\left[\begin{array}{c}
C \\
C A \\
\vdots \\
C A^{n-1}
\end{array}\right]
$$

En este documento se analiza la respuesta de diferentes tipos de estimadores de estado y su versatilidad para estimar variables en sistemas lineales. Se implementaron una serie de circuitos (sistemas embebidos) para el manejo de motores de corriente continua y a su vez se desarrolló software para su manipulación, el cual entrega como resultado de su operación los valores de las diferentes variables físicas de interés, correspondientes a la corriente del estator y la velocidad angular; estos valores luego serán comparados con los resultados obtenidos en los observadores de estado implementados.

\section{MATERIALES Y MÉTODOS}

\subsection{Modelo del motor de corriente continua}

Para abordar el diseño de los diferentes tipos de observadores de estados que se analizarán en este documento primero se debe obtener el modelo que describa correctamente la dinámica del proceso y, para lograr este objetivo, se hace una descripción fenomenológica de la dinámica del motor DC con base en su representación 
matemática [17]. Una vez cumplida esta etapa de descripción del sistema, se procede a realizar el análisis de observabilidad de tal manera que se cuente con los criterios mínimos necesarios para el diseño de los observadores de estado.

Para comenzar, en la figura (2) se muestra el circuito equivalente de un motor DC; a partir de este esquema se plantean las ecuaciones diferenciales que describen la dinámica del sistema, a saber [18]:

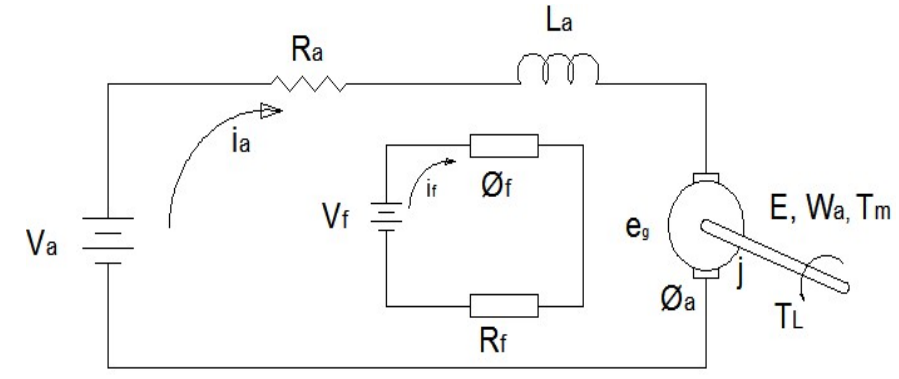

Figura 2. Circuito equivalente motor DC. Elaboración propia con base en [18].

$$
\begin{gathered}
R_{a} i_{a}+L_{a} \frac{d i a}{d t}+e_{k}=V_{a} \\
e_{g}=c_{1} \varphi \omega_{a} \\
T_{m}=C_{2} \varphi i i_{a} \\
R_{f} i_{f}+N_{f} \frac{d \varphi}{d t}=V_{f} \\
\varphi_{f}=f_{\left(i_{f}\right)} \\
\frac{d \varepsilon}{d t}=\omega_{a} \\
\left(J_{m}+J_{L}\right) \frac{d \omega a}{d t}=T_{m}-T_{L}-\beta \omega_{a}
\end{gathered}
$$

De donde,

- $R a$ : resistencia de armadura.

- Ia: corriente de armadura.

- La: inductancia de armadura.

- $e_{g}$ : voltaje inducido en la armadura.

- $V a$ : voltaje de armadura.

- $W a$ : velocidad angular.

- $\varphi f$ : flujo magnético.

- $C_{1}$ : constante de voltaje.

- $C_{2}$ : constante de torque.

- Jm: momento de inercia del motor.

- $J_{L}$ : momento de inercia de la carga.

- $T_{m}$ : torque del motor.

- $T_{L}$ : Torque de la carga.

- $R_{f}$ : Resistencia de campo.

- $i_{f}$ : Corriente de campo.

- $\quad N_{f}$ : Número de espiras bobinado de campo.

- $V_{f}$ : Voltaje de campo.

- $\varepsilon$ : Ángulo de rotación.

- $\beta$ : Coeficiente de fricción viscosa.

Las ecuaciones de estado para describir el modelo son:

$$
\begin{gathered}
\frac{d i_{a}}{d t}=-\frac{R_{a}}{L_{a}} i_{a}-\frac{K_{v}}{L_{a}} w_{a}+\frac{V_{a}}{L_{a}} \\
\frac{d w_{a}}{d t}=\frac{k_{v}}{J} i_{a}-\frac{\beta}{J} w_{a}-\frac{T_{L}}{J}
\end{gathered}
$$


Comparación de desempeño de observadores de estado en sistemas lineales con aplicación a un motor de corriente continua Performance comparison of state observers in linear systems through the application to a continuous current engine

La representación del sistema en espacio de estado debe tomar la forma descrita previamente en la ecuación (1). Para ello, las expresiones en (4) se pueden escribir de la siguiente forma:

$$
\begin{aligned}
& \dot{x}_{1=-\frac{R a}{L a} x_{1}-\frac{K_{v}}{L a} x_{2}+\frac{V_{a}}{L_{a}}} \\
& \dot{x}_{2=\frac{K_{v}}{J}} x_{1}-\frac{\beta}{J} x_{2}-\frac{T_{L}}{J}
\end{aligned}
$$

Donde las matrices de estado que representan la dinámica del sistema son:

$$
\begin{gathered}
{\left[\begin{array}{l}
\dot{x}_{1} \\
\dot{x}_{2}
\end{array}\right]=\left[\begin{array}{cc}
-\frac{R_{a}}{L_{a}} & -\frac{K_{v}}{L_{a}} \\
\frac{K_{v}}{J} & -\frac{\beta}{J}
\end{array}\right]\left[\begin{array}{l}
x_{1} \\
x_{2}
\end{array}\right]+\left[\begin{array}{c}
\frac{1}{L_{a}} \\
0
\end{array}\right]\left[\begin{array}{l}
V_{a} \\
T_{L}
\end{array}\right]} \\
y=\left[\begin{array}{ll}
1 & 0
\end{array}\right]\left[\begin{array}{l}
x_{1} \\
x_{2}
\end{array}\right]
\end{gathered}
$$

Las ecuaciones en (6) describen la representación de estados de un motor DC, modelo que será empleado en el diseño de observadores de las secciones subsiguientes.

\subsection{Observador de Luenberger}

Luenberger plantea en su artículo "Observing the State of a Linear System" [19] la definición de un observador de estados como un sistema dinámico que estima del vector de estados de un sistema en observación. Para el diseño de observadores es necesario verificar la condición de observabilidad, la cual es una condición no necesaria, pero sí primordial para el ajuste de las ganancias de los observadores. La observabilidad de un sistema se establece a partir de la siguiente definición [19]:

Definición: el sistema $(F, H)$ es observable si para cualquier estado de partida $x(0)$ existe un número $N$ finito, tal que $x(0)$ pueda ser calculado de la observación de $y(0), y(1), \ldots, y(N-1)$.

Una vez verificada esta condición de observabilidad procedemos a diseñar el observador Luenberger cuya estructura es:

$$
\begin{gathered}
\dot{\hat{x}}=A \hat{x}+B u+k_{c}(y-c \hat{x}) \\
\hat{y}=c \hat{x}
\end{gathered}
$$

Donde $\dot{\hat{x}}$ es la derivada de la estimación de los estados; $\hat{y}$ es la estimación de la variable de medición; y $k_{c}$ representa las ganancias del observador de estado.

\subsection{Filtro de Kalman}

El filtro de Kalman es un procedimiento matemático que opera por medio de un mecanismo de predicción y corrección. Este algoritmo pronostica el nuevo estado del sistema a partir de su estimación previa, añadiendo un término de corrección proporcional al error de predicción, de tal forma que este último es minimizado estadísticamente. Puede tener error en la estimación, requiere resolver la ecuación de Riccati y los estados iniciales deben comenzar cerca a los estados reales [13], [15]. A continuación, se describe la estructura del Filtro de Kalman lineal.

\section{Ecuación de estados}

Ecuación de salida:

$$
\begin{aligned}
: x_{k+1} & =A x_{k}+B u_{k}+w_{k} \\
y_{k} & =C x_{k}+v_{k}
\end{aligned}
$$

En la ecuación anterior, $A, B, C$ denotan matrices conocidas que describen el proceso; $k$ representa el instante discreto; $x$ son los estados del sistema; $u$ es el vector de entradas del sistema; $y$ constituye la medición de la salida; $w$ describe el ruido estocástico del proceso; y $v$ es el ruido estocástico en la medición. La estructura para el filtro de Kalman Lineal puede ser descrita de la siguiente manera:

$$
\begin{gathered}
K_{k}=P_{k} C^{T}\left(C P_{k} C^{T}+R\right)^{-1} \\
\hat{x}_{k+1}=\left(A \hat{x}_{k}+B u_{k}\right)+K_{k}\left(y_{k}-C \hat{x}_{k}\right) \\
P_{k+1}=A\left(I-K_{k} C\right) P_{k} A^{T}+Q
\end{gathered}
$$

Donde:

- $\hat{x}_{k}$ : estimación de $x_{k}$. 
- $k_{k}$ : matriz de ganancias de Kalman.

- $P_{k}$ : covarianza del error.

- Q: covarianza del ruido del proceso.

- $R$ : covarianza del ruido de la medición.

\subsection{Observador por modos deslizantes}

El desarrollo y la implementación de los observadores por modos deslizantes es una metodología que principalmente está enfocada a los sistemas y procesos no lineales [18], [20]. Para reconstruir los estados de un proceso no lineal, puede usarse un observador de estado no lineal de orden completo o de orden reducido, tal como:

Con

$$
\begin{gathered}
\dot{n}(t)=F(n(t), y(t), u(t)) ; \quad n(0)=n_{0} \\
\hat{x}(t)=H(n(t), y(t)
\end{gathered}
$$

$$
\begin{gathered}
\dot{x}(t)=f(x(t), u(t)), x(0)=x_{0} \\
y(t)=h(x(t))
\end{gathered}
$$

Donde $\hat{x}(t)$ : vector de variables de estado estimadas, $\|\hat{x}(t)-x(t)\| \rightarrow 0$ cuando $t \rightarrow \infty$, para todo proceso no lineal: Además, los términos $x=\left[x_{1} \ldots x_{n}\right]^{T} \in X, u=\left[u_{1} \ldots . u_{m}\right]^{T} \in U^{(0)}$ e $y=\left[y_{1} \ldots y_{q}\right]^{T}$ son vectores de estado, medición de la entrada y medición de la salida ya anteriormente definidos. La expresión de la ecuación (10) es de orden completo cuando $n \in R^{n}$. Este tipo de observador tiene grandes aplicaciones en sistemas altamente perturbados, dado que presenta gran insensibilidad a las señales de perturbación externas como un ruido de proceso [21]-[23]. La estructura para el observador por modos deslizantes se presenta a continuación:

$$
\begin{gathered}
\dot{\hat{x}}=A x+B u+\operatorname{Lsign}(y-c \hat{x}) \\
\hat{y}=c \hat{x}
\end{gathered}
$$

Donde:

- L: ganancia del observador.

- $L=\left[\begin{array}{l}L_{1} \\ L_{2}\end{array}\right]$

- $L_{1}$ : ganancia para la manipulación de la amplitud del castañeo (chattering).

- $L_{2}$ : ganancia que depende de la dinámica del proceso.

Tabla 1. Parámetros del motor.

\begin{tabular}{|c|l|l|}
\hline Parámetro & \multicolumn{1}{|c|}{ Valor } & \multicolumn{1}{|c|}{ Unidad } \\
\hline$R_{a}$ & 4.5 & Ohmios \\
\hline$L_{a}$ & 0.5837 & $\mathrm{H}$ \\
\hline$J$ & 0.00001 & $\mathrm{Kg}^{*} \mathrm{~m}^{2}$ \\
\hline$B$ & 0.00026 & $\mathrm{~N}^{*} \mathrm{~m}^{*} \mathrm{~s} / \mathrm{rad}$ \\
\hline$K_{v}$ & 0.087 & $\mathrm{~V}^{*} \mathrm{~s} / \mathrm{rad}$ \\
\hline
\end{tabular}

\section{RESULTADOS DE LA APLICACIÓN DE LOS OBSERVADORES}

En esta sección se muestran los resultados de validación del modelo de motor DC presentado en las secciones anteriores y la simulación del desempeño de observadores Luenberger, Kalman y por modos deslizantes para el mismo modelo. Los parámetros de simulación se describen en la tabla 1. La figura 3 corresponde a la respuesta de la corriente estatórica del motor cuando es sometido a una excitación de tipo escalón y el sistema se encuentra en lazo abierto. 
Comparación de desempeño de observadores de estado en sistemas lineales con aplicación a un motor de corriente continua Performance comparison of state observers in linear systems through the application to a continuous current engine

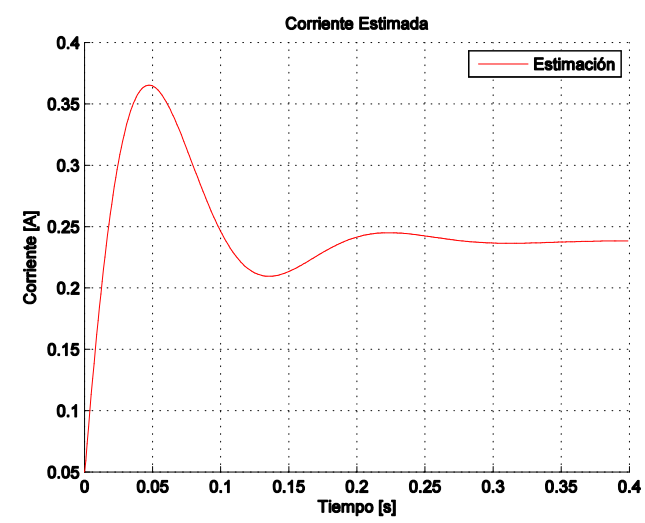

Figura 3. Dinámica de la corriente estatórica del motor utilizando el modelo de estados.

\subsection{Resultados de Observador Tipo Luenberger}

La figura 4 corresponde a la implementación del algoritmo de observación de estado de Luenberger, en esta figura se percibe el comportamiento de la señal de la variable en observación (velocidad del motor); para este proceso de estimación se usan como parámetros la medición de la corriente estatórica del motor y el modelo del sistema, información necesaria para la realización del proceso de estimación de estados.

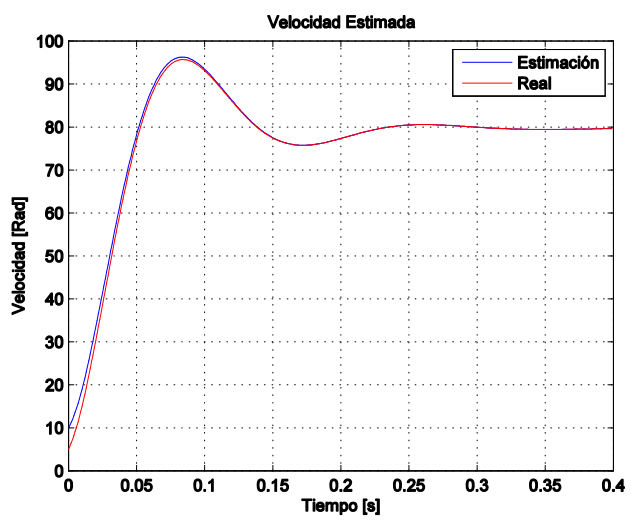

Figura 4. Dinámica de la velocidad según el modelo (rojo) y el desempeño del observador de Luenberger para el sistema (azul).

En la figura (4) se muestra la respuesta de la señal de velocidad del motor cuando es excitado por una señal de tipo escalón, en esta imagen se observa que la señal estimada converge a los valores de la señal real del sistema. Esto se logra gracias a que el observador Luenberger estima los estados del sistema indicado basado en la medición de la entrada, la salida y el modelo del sistema.

\subsection{Resultados de observador por Filtro de Kalman}

La figura (5) corresponde a la respuesta de la corriente estatórica del motor cuando este es sometido a una excitación de tipo escalón y el sistema se encuentra en lazo abierto, en esta imagen se observa que la medición presenta ruido, el cual puede ser ocasionado por el elemento sensor $y$, a su vez, este puede ocasionar problemas en la acción de control del proceso debido a las oscilaciones de la señal. En este trabajo se utiliza el Filtro de Kalman Bucy como estimador de estado, de tal manera que se mide la corriente estatórica del motor DC, y se estima la velocidad angular de dicho motor, además de contar con la posibilidad de determinar todos los estados del sistema [24]. 


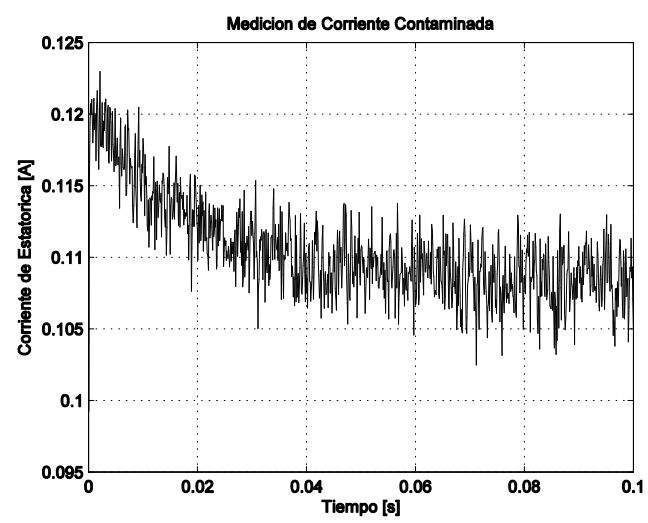

Figura 5. Dinámica de la corriente estatórica en presencia de ruido de medición.

La figura (6) muestra la respuesta real del motor y la respuesta del observador de estados cuando el motor es sometido a una excitación; en esta imagen se observa que la señal real medida del motor presenta oscilaciones generalmente debidas al elemento sensor o inherentes a la dinámica del proceso, adicional a esto se observa la efectividad del filtro de Kalman a la hora de obtener señales de buenas características de estabilidad, partiendo de señales altamente contaminadas. También se puede ver que la señal del observador converge a la señal real y que es más estable.

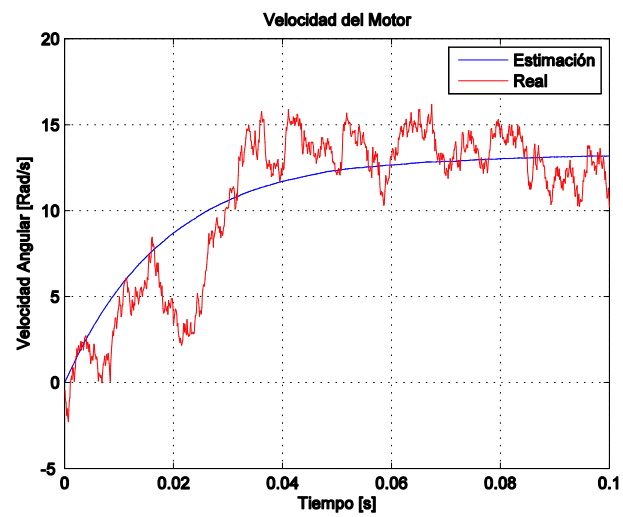

Figura 6. Dinámica de la velocidad cuando hay ruido de medición (rojo) y respuesta utilizando Filtro de Kalman (azul).

\subsection{Resultados de observador por Modos Deslizantes}

En la figura (7) se advierte que la señal de velocidad estimada del motor converge hacia a la señal de velocidad real, este es el momento en el cual el observador de estado alcanza la superficie deslizante que hace que la señal del observador converja a la señal real del sistema. Además de esto también se puede apreciar que este observador de estados por modos deslizantes presenta mayor velocidad de convergencia que los observadores anteriormente implementados. Cabe anotar que una falencia que posee este tipo de observador es el fenómeno de chattering o zigzagueo de las señales estimadas, que se da una vez el observador alcanza la superficie deslizante. 
Comparación de desempeño de observadores de estado en sistemas lineales con aplicación a un motor de corriente continua Performance comparison of state observers in linear systems through the application to a continuous current engine

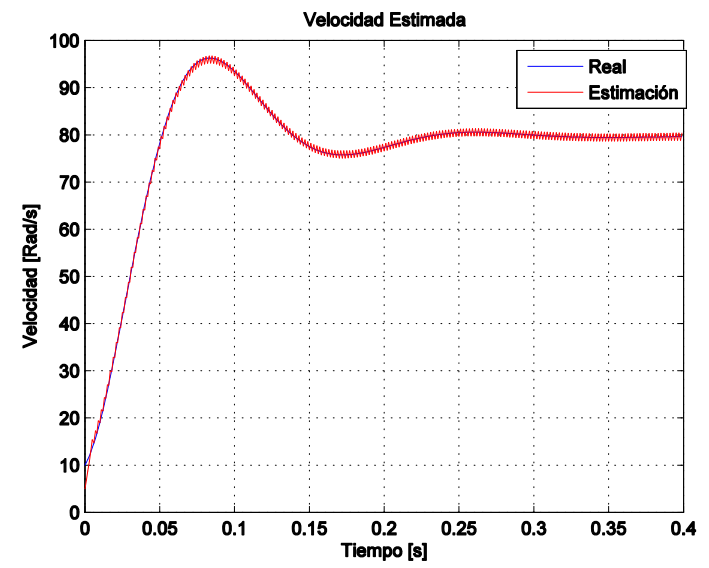

Figura 7. Dinámica de la velocidad según el modelo (azul) y respuesta del observador por modos deslizantes (azul).

\section{CONCLUSIONES}

En este trabajo se evidencia que es posible reconstruir el vector de estados de un sistema físico a partir de su modelo matemático y de las mediciones de sus entradas y salidas utilizando herramientas como observadores de estado. El sistema analizado fue un motor de corriente continua. En general, puede decirse que la estimación de la salida de un proceso puede llegar a ser la solución para la medición de variables que son difícilmente medibles o simplemente no pueden medirse.

En cuanto el desempeño de las diferentes estrategias implementadas, se resalta que el observador Luenberger puede ser utilizado en procesos en los que existe un bajo nivel de ruido y su velocidad de convergencia no sea crítica; esto puede interpretarse como que este tipo de observador es útil para aquellos procesos que se puedan tener en ambientes controlados. Se observa que el filtro de Kalman es una herramienta muy efectiva y confiable para la estimación de señales altamente contaminadas, puede soportar procesos con señales muy ruidosas y entregar una señal estimada limpia de dicho proceso. Por último, el observador por modos deslizantes presenta muy buena velocidad de convergencia y un error en estado estable igual a cero, esto debido a la superficie deslizante y su inmunidad a ruidos o perturbaciones que actúan sobre el sistema, pero una falencia que posee es el chattering, que se presenta una vez el observador encuentra la superficie deslizante.

A partir de los resultados puede decirse que no hay un observador perfecto que se desempeñe satisfactoriamente en cualquier condición de operación. Según la aplicación o las características del proceso se puede seleccionar un observador que sea el ideal para cada sistema, dado que, como se pudo evidenciar en la respuesta de los observadores, cada uno de ellos tiene su fortaleza específica.

\section{REFERENCIAS}

[1] A. Navarro, O. Begovich, J. D. Sanchez-Torres, G. Besancon, and J. A. P. Murillo, "Leak detection and isolation using an observer based on robust sliding mode differentiators," in World Automation Congress (WAC), 2012, 2012, pp. 1-6.

[2] J. D. Sánchez-Torres, A. G. Loukianov, J. A. P. Murillo, B. Giraldo, and H. Botero, "A high order sliding mode observer for systems in triangular input observer form," in Robotics Symposium, 2011 IEEE IX Latin American and IEEE Colombian Conference on Automatic Control and Industry Applications (LARC), 2011, pp. 16.

[3] J. D. Sanchez-Torres, A. G. Loukianov, J. A. Murillo, B. Giraldo, and H. Botero, "An equivalent control based second order sliding mode observer using robust differentiators," in Robotics Symposium, 2011 IEEE IX Latin American and IEEE Colombian Conference on Automatic Control and Industry Applications (LARC), Bogotá, Colombia, 2011, pp. 1-6.

[4] J. E. Castano, J. A. Patino, and J. J. Espinosa, "Model identification for control of a distillation column," in Robotics Symposium, 2011 IEEE IX Latin American and IEEE Colombian Conference on Automatic Control and Industry Applications (LARC), 2011, pp. 1-5.

[5] M. Giraldo and J. Patiño, "Controladores prealimentados y realimentados para sistemas de primer orden con tiempo muerto y ganacia variable," Rev. CINTEX, vol. 16, pp. 103-108, 2011.

[6] P. Deossa, J. Patino, J. Espinosa, and F. Valencia, "A comparison of Extended Kalman Filter and Levenberg-Marquardt methods for neural network training," in Robotics Symposium, 2011 IEEE IX Latin American 
and IEEE Colombian Conference on Automatic Control and Industry Applications (LARC), Bogotá, Colombia, 2011, pp. 1-5.

[7] F. Valencia, J. López, J. Patiño, and J. Espinosa, "Bargaining Game Based Distributed MPC," in Distributed Model Predictive Control Made Easy, Springer Netherlands, 2014, pp. 41-56.

[8] P. Deossa, A. Marquez, and J. Espinosa, "Integration of economic MPC, energy load and price estimation with Holt Winters models," in 2014 IEEE PES Transmission \& Distribution Conference and Exposition - Latin America (PES T\&D-LA), Medellin, Colombia, 2014, pp. 1-5.

[9] A. E. Florián Villa, "Metodología de análisis de observabilidad para estimadores de estado en sistemas de energía eléctrica," Master thesis, Universidad Nacional de Colombia, Colombia, 2015.

[10] R. Horta, J. Espinosa, and J. Patino, "Frequency and voltage control of a power system with information about grid topology," in Automatic Control (CCAC), 2015 IEEE 2nd Colombian Conference on, 2015, pp. 1-6.

[11] V. Srdanovic, O. B. Henao, and S. H. Ruiz, "Modelado e Identificación de un Sistema Electromecánico y Diseño del Control PID para Gobernar Inalámbricamente el Desplazamiento de un Objeto Móvil," Rev. CINTEX, vol. 22, no. 1, pp. 25-46, 2017.

[12] Julian Alberto Patino, J. J. Espinosa, and R. E. Correa, "A comparison of Kalman-based schemes for localization and tracking in sensor systems," in Communications (LATINCOM), 2010 IEEE Latin-American Conference on, 2010, pp. 1-5.

[13] J. A. Patiño Murillo, "Kalman-based schemes for mobile nodes localization in ad-hoc networks," Universidad Nacional de Colombia - Sede Manizales, 2011.

[14] A. Navarro, J. D. Sanchez-Torres, O. Begovich, G. Besancon, and J. Patino-Murillo, "An algebraic observer for Leak Detection and Isolation in plastic pipelines," in Control Conference (ECC), 2013 European, Zurich, Switzerland, 2013, pp. 926-931.

[15] H. A. Botero Castro and H. D. Alvarez Zapata, "Una revisión de los métodos más frecuentes para la estimación del estado en procesos químicos," Dyna, no. 158, pp. 135-146, 2009.

[16] R. E. Kalman, "A New Approach to Linear Filtering and Prediction Problems," Trans. ASME-Journal Basic Eng., vol. 82, no. Series D, pp. 35-45, 1960.

[17] B. Osorio, J. Osorio, L. Mejía, G. E. Campillo, and R. Covaleda, "La conceptualización del campo eléctrico y magnético. Análisis de las concepciones de los estudiantes," Rev. CINTEX, vol. 20, no. 1, pp. 123-137, 2015.

[18] G. Rubio-Astorga, J. D. Sánchez-Torres, J. Cañedo, and A. G. Loukianov, "High-Order Sliding Mode Block Control of Single-Phase Induction Motor," IEEE Trans. Control Syst. Technol., vol. 22, no. 5, pp. 1828-1836, Sep. 2014.

[19] D. G. Luenberger, "Observing the State of a Linear System," IEEE Trans. Mil. Electron., vol. 8, no. 2, pp. 74-80, Apr. 1964.

[20] S. V. Drakunov and V. I. Utkin, "Sliding mode control in dynamic systems," Int. J. Control, vol. 55, no. 4, pp. 1029-1037, Apr. 1992.

[21] J. Barrientos and H. Botero, "Diseño de un observador de estado en un bioproceso para la producción de etanol," Rev. CINTEX, vol. 15, pp. 27-35, 2010.

[22] J. Patino, F. Valencia, and J. Espinosa, "Sensitivity analysis for frequency regulation in a two-area power system," Int. J. Renew. Energy Res., vol. 7, no. 2, pp. 700-706, 2017.

[23] J. Patiño, J. D. López, and J. Espinosa, "Analysis of Control Sensitivity Functions for Power System Frequency Regulation," in Applied Computer Sciences in Engineering, vol. 915, J. C. Figueroa-García, E. R. López-Santana, and J. I. Rodriguez-Molano, Eds. Cham: Springer International Publishing, 2018, pp. 606-617.

[24] Z. Yin, C. Zhao, Y.-R. Zhong, and J. Liu, "Research on Robust Performance of Speed-Sensorless Vector Control for the Induction Motor Using an Interfacing Multiple-Model Extended Kalman Filter," IEEE Trans. Power Electron., vol. 29, no. 6, pp. 3011-3019, Jun. 2014. 